mycophenolate mofetil, cyclophosphamide, and stem cell transplantation. In the first round, patients were asked to indicate which information on the benefits and harms is essential for making a treatment decision; they could also add new items. In the second and third rounds, patients were asked to prioritize the items. After the third round, a live, online panel discussion was held in order to reach consensus. Consensus was defined as $\geq 75 \%$ agreement among panel members.

Results: Of the 36 patients invited, $67 \%(n=24)$ completed the first, $69 \%(n=25)$ the second and $75 \%(n=27)$ the third round. In the third round, median age of participants was 51 years (IQR 18), median disease duration was 4 years (IQR 5), $52 \%$ was female. Most patients had completed either an intermediate education level $(59 \%)$ or high level of education $(30 \%)$. Patients were or had been treated with mycophenolate mofetil $(67 \%)$, methotrexate $(44 \%)$, cyclophosphamide $(41 \%)$, autologous stem cell transplantation (26\%), rituximab $(4 \%)$. Seven percent of patients were treatment-naïve. After the third round, eight patients joined the online panel discussion to reach final consensus. The panel reached consensus on seven benefits (prolonged progression-free survival, improved quality of life, improved daily functioning, improved pulmonary function, improved skin thickness, improved mobility and reduced fatigue) and four adverse events (treatment-related mortality, viral infections, cardiac damage, increased risk of cancer) as key themes for the decision making process (Table 1).

Conclusion: This study identified essential aspects of the four main treatment options for dcSSc. Our results can be used to develop effective patient information.

Table 1. Agreement on benefits and harms that should be addressed

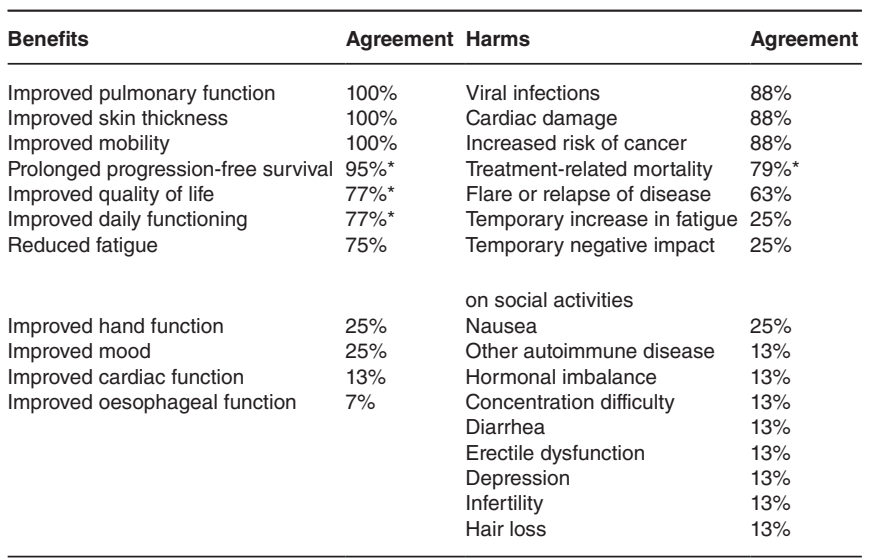

*Items on which consensus was reached in round 3

Disclosure of Interests: None declared

DOI: 10.1136/annrheumdis-2021-eular.396

\section{AB0915-PARE DEVELOPMENT AND USABILITY OF A WEB-BASED PATIENT-TAILORED TOOL TO SUPPORT ADHERENCE TO URATE-LOWERING THERAPY IN GOUT PATIENTS}

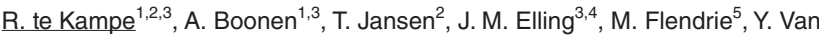
Eijk-Hustings ${ }^{1}$, M. Janssen ${ }^{2}$, C. Van Durme ${ }^{1}$, H. De Vries ${ }^{3,4} .{ }^{1}$ Maastricht University Medical Center, Internal Medicine, Maastricht, Netherlands; ${ }^{2}$ VieCuri Medical Center, Rheumatology, Venlo, Netherlands; ${ }^{3}$ Maastricht University, Care and Public Health Research Institute (CAPHRI), Maastricht, Netherlands; ${ }^{4}$ Maastricht University, Health Promotion, Maastricht, Netherlands; ${ }^{5}$ Radboud University Nijmegen Medical Centre, Rheumatology, Nijmegen, Netherlands

Background: Adherence to prescribed urate-lowering therapy (ULT) among gout patients is considered to be among the poorest of all chronic conditions. eHealth programs can be a possible opportunity to foster ULT adherence.

Objectives: This study describes the development and usability evaluation of a web-based tool to support ULT adherence among gout patients, specifically designed for a complement to usual care.

Methods: The Integrated Change (I-Change) model was used as theoretical basis for the development. The model combines various socio-cognitive theories and differentiates between three phases: a pre-motivational, a motivational, and a post-motivational phase. In practices, the I-Change gout tool contains three sessions, following the three phases of the I-Change model. Patients receive tailored feedback based on their answers in the form of animated videos and text messages after each session, and are prompted to set specific goals and action plans for their ULT adherence. The content and development of the I-Change gout tool was determined along an iterative process within a steering group of clinicians and researchers, supported by patient interviews and gout specific literature related to key aspects of medication adherence behavior. A cross-sectional mixed methods design was used to test usability of the support tool consisting of a think aloud method and a usability questionnaire.

Results: The steering group decided on the content of the three sessions of the I-Change gout tool. Depending on the intention to change ULT adherence behavior patients were navigated through the I-Change gout tool, patients with a low intention go through all 3 sessions and patients with a high intention go through the pre- and post-motivational session (figure 1). In total, the I-Change gout tool contains three sessions with 80 questions, 66 tailored textual feedback messages, and 40 tailored animated videos.

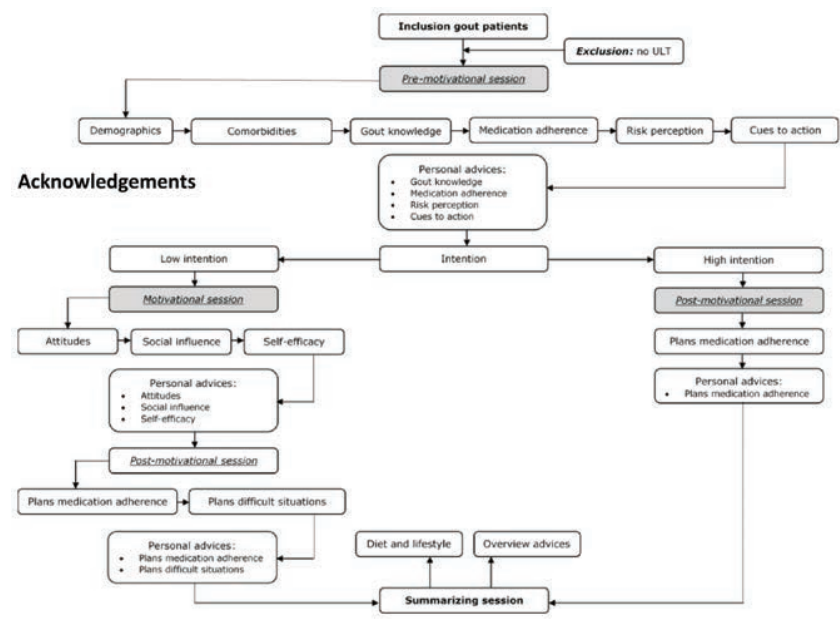

Figure 1. Flowchart of the computer-tailored I-Change gout tool for urate-lowering therapy adherence.

Twenty gout patients and seven healthcare professionals participated in the usability tests. The program end score rating for the gout tool was on average 8.4 \pm 0.9 (range 6-10) for patients and 7.7 \pm 1.0 (range 6-9) for healthcare professionals. Furthermore, participants reported a high intention to use and/or recommend the program in the future. Yet, participants identified some issues for further improvement of the systems user-friendliness by addressing barriers (e.g. more explicitly navigation) and weaknesses (e.g. technical and health literacy) The I-Change gout tool was updated according suggestions of improvements of the participants.

Conclusion: This study provides initial support for the usability by patients and healthcare professionals of a ULT adherence I-Change gout tool. Further studies need to be conducted to assess its efficacy and (cost-) effectiveness in daily practice.

Disclosure of Interests: None declared

DOI: 10.1136/annrheumdis-2021-eular.1500

\section{AB0916-PARE KNOWLEDGE OF DISEASE, DIAGNOSIS, ADHERENCE AND IMPACT OF RESEARCH IN PATIENTS WITH INFLAMMATORY ARTHRITIS}

M. Canavan ${ }^{1,2}$, V. Marzaioli ${ }^{1,2}$, A. Donnelly ${ }^{3}$, S. Wade ${ }^{1,2}$, A. Fraser ${ }^{4}$, T. O'sullivan ${ }^{3}$, S. Harney ${ }^{5}$, A. Ireland ${ }^{6}$, D. Veale ${ }^{2}$, U. Fearon ${ }^{1,2} .{ }^{1}$ Trinity College Dublin, Molecular Rheumatology, Dublin, Ireland; ${ }^{2}$ St Vincent's University Hospital, Centre for Arthritis and Rheumatic Diseases, Dublin, Ireland; ${ }^{3}$ Patient Advocate, Patient Advocate, Dublin, Ireland; ${ }^{4}$ University of Limerick, UCL, Department of Rheumatology, Limerick, Ireland; ${ }^{5}$ Cork University Hospital, UCC Department of Rheumatology, Cork, Ireland; ${ }^{6}$ Clanwilliam Square, Grand Canal Dock, Dublin, Ireland

Background: The diagnosis and treatment of inflammatory arthritis has been transformed largely by the development of drugs that target specific molecules of the immune system. However, these changes have increased the complexity of the mechanisms of disease, its treatment and patients understanding. Patient education is needed in this area to facilitate decision making and to facilitate strong patient-partnerships in research.

Objectives: The aim of this study was to examine the level of understanding of inflammatory arthritis patients and the need for strong patient-partnership in research.

Methods: An online anonymous survey addressed questions about diagnosis, routine tests, medications and how they work, medication adherence, disease flare, heredity, pregnancy, and patient involvement in research.

Results: There were 1,873 respondents, 1416 of which had inflammatory arthritis (IA)-RA (65.8\%) and PsA (34.2\%). They were predominantly female (RA $86 \%$ 